\title{
Médecin de dernier recours
}

\section{Jean Martin}

Dr med., membre de la rédaction

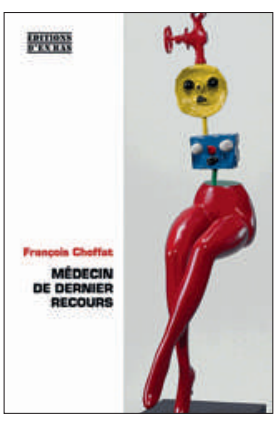

François Choffat Médecin de dernier recours

Lausanne: Editions d'en bas; 2015.

292 pages. $34 \mathrm{CHF}$. ISBN 978-2-829-00510-7

jean.martin[at]saez.ch
Le Docteur François Choffat est un médecin connu de Suisse romande. Lui et moi nous connaissons de longue date et partageons d'avoir tous deux travaillé en début de carrière dans des pays en développement - le Maroc pour lui, d'où avec son épouse il a rapporté un livre attachant, Sur les traces d'Ounamir. Après avoir été assistant dans des hôpitaux romands et travaillé outremer, l'auteur s'est installé comme généraliste au bord du lac de Neuchâtel, près de la frontière Vaud-Fribourg. Sa curiosité de paradigmes médicaux autres (y compris guérisseurs et "panseurs de secret» dans le Jura) et des expériences positives dans des situations où l'allopathie s'avérait décevante l'ont amené à s'attacher à l'homéopathie, devenue une partie majeure de sa pratique. Il s'est aussi beaucoup préoccupé d'alimentation, en étant un disciple de Catherine Kousmine, et a fondé un Centre de santé holistique dont il raconte les péripéties parfois difficiles.

«Le chamanisme et la chirurgie sont les symboles de deux pratiques diamétralement opposées de l'art de guérir... un pôle humaniste et un pôle mécaniste. Pour moi, ces deux pôles sont devenus indissociables, et complémentaires comme le cerveau gauche et le cerveau droit [...] Dans ma pratique, il y a d'un côté l'héritage revendiqué de la médecine conventionnelle, de l'autre certaines médecines complémentaires. Cette synthèse a été le fruit d'une longue confrontation entre mes croyances initiales et les échecs que la réalité m’a infligés.» "L’homéopathie m’a fait redécouvrir la réalité d'une certaine Energie vitale, qui échappe à la démarche scientifique et la complète.»

C'est toute une trajectoire que retrace Médecin de dernier recours, titre lié au fait que, pas rarement, des patients se sont adressés à lui après avoir cherché du secours ailleurs, en particulier dans la médecine orthodoxe, sans qu'un remède soit trouvé. Choffat s'attache à décrire ses débuts en homéopathie, ce qu'il a appris de cette méthode et la manière de la pratiquer, ainsi que d'autres démarches dans lesquelles il s'est formé, en Suisse et à l'étranger. L'ouvrage fourmille de vignettes cliniques illustrant le propos. L'espace ne permet pas de faire la liste des sujets traités, dont des critiques marquées à l'endroit d'aspects commerciaux de la médecine. Il consacre un chapitre à ses réserves vis-à-vis des pratiques vaccinales qu'il juge trop systématiques et influencées par l'industrie. Un autre est dédié à la sclérose en plaques, une des "causes perdues» pour lesquelles on faisait appel à lui.

A propos de EBM: «Le médecin devrait tempérer la dictature des statistiques par le bon sens, en fait par l'évidence du patient qui recourt à ses soins, son vécu, son témoignage.» A son dernier chapitre, l'auteur relève avoir toujours été captivé par les physiciens, mentionnant une demi-douzaine de grands noms et leurs intuitions: «Ces mystères nourrissent ma méditation et m’imprègnent de la transcendance de la Vie. Dans leur sillage, je suis au carrefour de la poésie, de l'humour, de la métaphysique [...] Ces physiciens connaissent la profondeur de leur ignorance. Leur façon de penser questionne notre médecine institutionnelle et son rationalisme [...] Face à l'exubérance de la recherche dans les sciences de la matière, je suis atterré en voyant des savants qui refusent de s'émerveiller devant autre chose que leur propre savoir..."

Les attitudes ont passablement changé à l'endroit des méthodes qu'on ne souhaite plus appeler parallèles ou alternatives mais complémentaires - pour ne pas faire penser à une confrontation inévitable. Aujourd'hui, il me paraît que beaucoup peuvent se dire d'accord avec F. Choffat que «le fait d'affirmer qu'il n'y a pas de salut en dehors de la médecine officielle relève de l'arrogance». Cela étant, on ne sera bien sûr pas toujours d'accord avec l'auteur dans ses affirmations. Mais on ne saurait nier son ouverture aux choses "autres», sa sincérité et son engagement. Ni sa volonté de tirer profit du potentiel de la médecine enseignée à la Faculté comme de méthodes dont les effets ne sont aujourd'hui pas expliqués. Ce livre est un exemple d'efforts tout à fait estimables, par des médecins au terme d'une carrière bien remplie, de rassembler vécu, leçons tirées, questionnements, sous une forme aisément accessible à d'autres. Ce récit de vie, structuré en sections faciles à consulter, pourra intéresser les professionnels de santé aussi bien qu'un large public. 